\title{
Minimum Emission Current for a Liquid Metal Ion Source
}

\author{
Richard G. Forbes and Vasily G. Suvorov \\ University of Surrey, Advanced Technology Institute (BB), \\ School of Electronics and Physical Sciences, Guildford, Surrey GU2 7XH, UK.
}

It is well known experimentally that a liquid-metal ion source (LMIS) has a minimum stable emission current, typically around 1 to $2 \mu \mathrm{A}$. Attempts to run at lower current result in instability, or current extinction due to collapse of the liquid-metal cone (the "Taylor-Gilbert cone") that is essential to LMIS operation. The existence of a minimum current seems to be one of the factors that limit our ability to improve the resolution of a Focused Ion Beam machine by reducing the emission current and the spot size at the specimen. So there is interest in having good understanding of the origin of minimum current, and of relevant theory able to predict its size and dependence on relevant parameters.

We have recently looked again at the existing theory of LMIS minimum current, developed by Beckman and others [1], and have discovered two theoretical difficulties with it. First, these authors assume that the emitter shape at low emission currents is a rounded Taylor cone. However, HVEM observations established long ago that at higher emission currents the emitter has the shape of 'a cusp on a cone'. Because there are electrostatic difficulties with the rounded Taylor cone assumption, it is theoretically probable that it also has this shape at lower currents.

Second, it is well established that the ion emission process in the LMIS is "field evaporation (FEV)". This is also the emission process in the Atom-Probe Field Ion Microscope. It is know from basic work long ago, in the context of field ion microscopy, that the variant of FEV theory used in Beckman's minimum-current theory contains a disabling mathematical error.

We have therefore reformulated minimum-current theory, for a LMIS with the shape of a cusp on a cone, using a FEV theory [2] that is known to be an approximation but which does often explain field evaporation results at low temperatures. Unfortunately, this theory does not yield plausible results for LMIS minimum current; the exact reasons for this are not currently understood. We have therefore reformulated FEV theory more generally, in terms of a second-order Taylor expansion of the FEV activation energy $Q$ about the field $F$ e at which $Q$ becomes zero. Best existing FEV theory has the first-order Taylor coefficient zero, but it seems clear that to get a plausible theory of LMIS minimum current it is necessary to have a first-order Taylor coefficient that is significantly large.

In unpublished work with D N Zurlev, one of us (RGF) has explored whether ion tunneling effects might give rise to a significant first-order Taylor coefficient in FEV theory, but we can find no convincing evidence of this. Further, no obvious cause of a significant first-order Taylor coefficient has yet emerged in the course of a re-exploration of the (simplified) analytical theory of the chargeexchange mechanism of FEV developed by one of us (RGF) some years ago (see Ref. [2]).

A further possibility that remains to be explored in detail is that, at low emission currents, the shape of the LMIS emitter corresponds neither to a 'cusp on a cone' nor to a 'rounded Taylor cone', but to 
some other shape that has an effective area of emission different from that assumed in 'cusp on a cone' model. It would also be helpful to develop full quantum-mechanical treatments of FEV for materials of relevance to LMIS applications, in particular gallium.

Notwithstanding these circumstances, it has been possible to develop an analytical theory [3] of minimum current that can explain minimum currents of the size typically observed (although these sizes cannot be predicted a priori, at present). The outlines of such a treatment are set out in Fig. 1.

\section{References}

[1] J. C. Beckman et al., J. Vac. Sci. Tech. B15 (1997) 2332.

[2] R.G. Forbes, Appl. Surface Sci. 87/88 (1995) 1.

[3] V.G. Suvorov and R.G. Forbes, Microelectron. Eng. 73-74 (2004) 126.

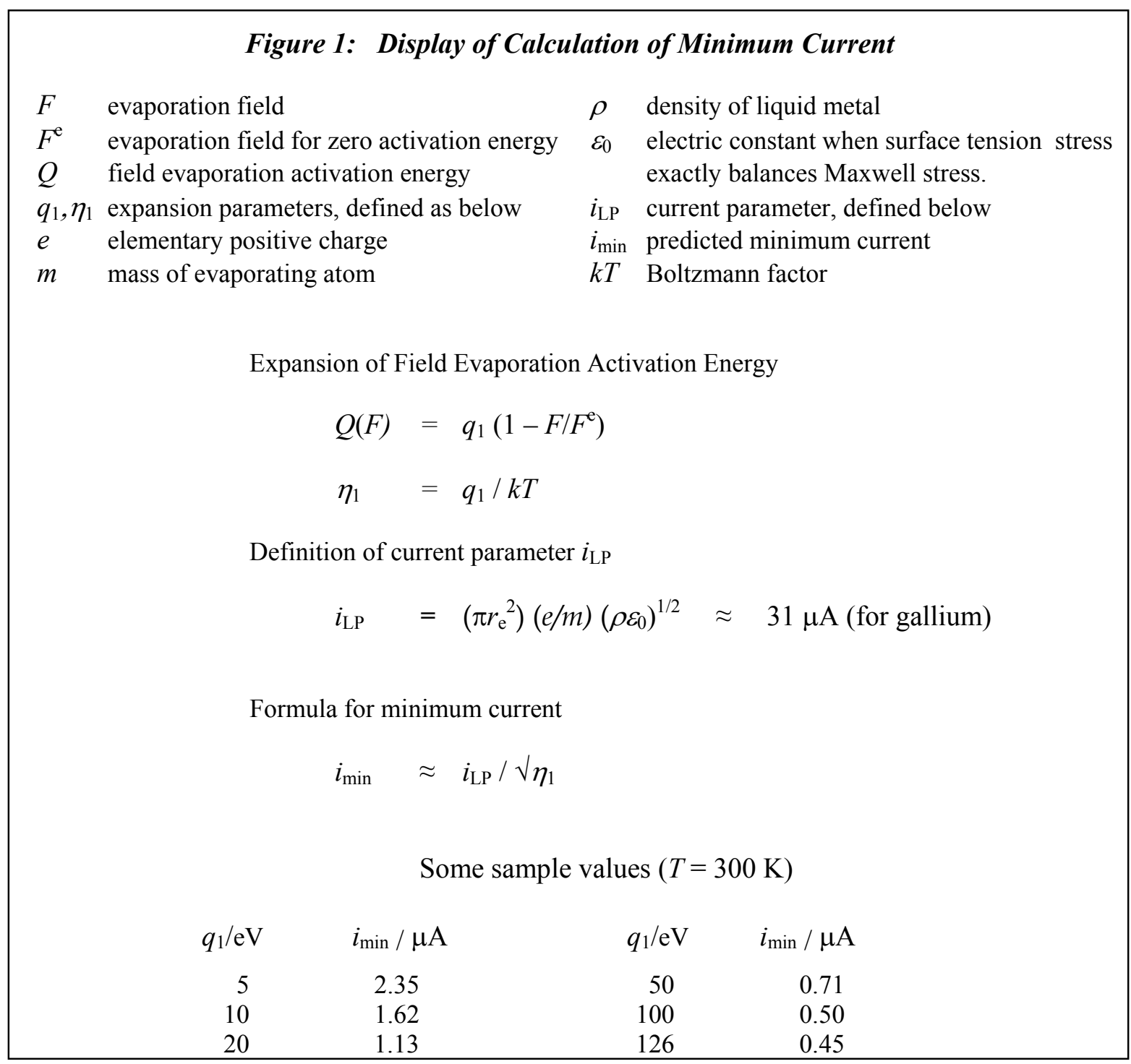

